\title{
Diferentes quantidades de ração préinicial para aves
}

\author{
Sangalli, G.G.; Nunes, R.V.; Broch, J. ${ }^{\circledR}$; Souza, C. e Eyng, C. \\ Universidade Estadual do Oeste do Paraná. Centro de Ciências Agrárias. Marechal Cândido Rondon. Paraná. Brasil.
}

PALAVRAS CHAVE ADICIONAIS

Avicultura.

Desempenho.

Morfometria intestinal.

Nutricao.

\begin{abstract}
RESUMO
O experimento teve como objetivo avaliar o desempenho, peso relativo dos órgãos do trato gastrointestinal e morfometria do jejuno de frangos de corte aos 21 dias, alimentados com diferentes quantidades de ração préinicial. Foram utilizados 576 pintos de corte distribuídos em um delineamento experimental inteiramente casualizado, contendo quatro tratamentos, que consistiram do fornecimento de diferentes quantidades de ração préinicial (100, 200, 300 e 400 gramas), com seis repetições. Ao término do consumo das respectivas quantidades de ração, as aves de cada unidade experimental passaram a receber uma ração inicial única, a base de milho e farelo de soja, até os 21 dias de idade. Os resultados obtidos foram submetidos à análise de variância e regressão polinomial ao nível de $5 \%$ de probabilidade. Houve efeito linear crescente $(\mathrm{P}<0,05)$ para conversão alimentar, havendo piora na conversão conforme aumento da quantidade de ração préinicial fornecida. O fornecimento de 100 gramas de ração préinicial proporcionou melhor conversão alimentar aos 21 dias de idade, sem interferir no desenvolvimento do trato gastrointestinal das aves.
\end{abstract}

\section{Pre starter broiler nutrition}

\section{SUMMARY}

\section{ADDITIONAL KEYWORDS}

Poultry science.

Performance.

Intestinal morphology.

Nutrition.

\section{INFORMATION}

\section{Cronología del artículo.}

Recibido/Received: 15.05.2019

Aceptado/Accepted: 05.03.2020

On-line: 15.04 .2020

Correspondencia a los autores/Contact e-mail:

brochjomara@yahoo.com.br

\begin{abstract}
The experiment aimed to evaluate the performance, relative weight of the organs of the gastrointestinal tract and morphometry of the jejunum of broilers at 21 days, fed with different amounts of prestarter diet. 576 broiler chick were assigned in a completely randomized experimental design to four treatments, which consisted of providing different amounts of prestarter diet (100,200,300 and 400 grams), and six replications and 24 birds per experimental unit. At the end of the consumption of the respective quantities of feed the birds in each experimental unit began to receive a ration based on corn and soybean meal, until the 21 days of age. The results were submitted to analysis of variance and polynomial regression to the $5 \%$ level of probability. The feed conversion rate linearly increased $(\mathrm{P}<0.05)$ with increasing the quantity of prestarter ration. The supply of 100 grams of prestarter ration provided better feed conversion to 21 days of age, without interfering in the development of gastrointestinal tract of birds.
\end{abstract}

\section{INTRODUÇÃO}

Compreender os fatores que determinam o crescimento e o desenvolvimento dos tecidos das aves é essencial para adequar os programas nutricionais, genéticos e de ambiência, bem como para definir a idade de abate para se obter melhor qualidade e rendimento de carne produzida.

No âmbito da nutrição, o uso de dietas pré-iniciais aliado a crescente evolução da avicultura, motivaram pesquisas nesta área, considerando as mudanças morfo-fisiológicas decorrentes no período pós-eclosão e a importância da qualidade nutricional das dietas fornecidas às aves.
Durante as últimas 72 horas de incubação, o embrião passa por diversas transformações, sendo a maior delas a maturação do trato gastrintestinal. Após três a cinco dias pós-eclosão a eficiência digestiva se desenvolve de forma rápida e neste período as reservas nutricionais e imunológicas do saco vitelino cessam, tornando-se exclusivo o fornecimento de nutrientes de fontes exógenas para sobrevivência da ave.

Após a eclosão ocorre a fase de transição da vida embrionária para a vida independente, na qual, o rápido acesso a alimentos exógenos é essencial (Noy e Sklan, 1998). Assim, nos primeiros dias de vida das aves, se faz necessário o fornecimento de uma dieta diferenciada, pois na primeira semana, as aves ainda 
não estão totalmente adaptadas aos processos digestivos; pois a capacidade funcional do sistema digestorio ainda não permite a adequada digestão e absorção de todos os nutrientes (dos Santos et al., 2016).

Durante a primeira semana pós-eclosão a alimentação precoce e uma rápida adaptação possuem efeito fundamental no desenvolvimento e crescimento dos pintinhos de corte. $\mathrm{O}$ fornecimento precoce de nutrientes aos pintinhos é essencial para aumentar a atividade mecânica intestinal, promover um rápido desenvolvimento intestinal e imonologico, permitindo assim uma maior assimilação da ração acarretando na melhora do desempenho geral das aves (Prabakar et al., 2016).

Para a formulação da primeira dieta a ser recebida pelos pintinhos desde o alojamento, deve-se considerar as características nutricionais diferenciadas dos animais nesta fase, sendo essencial que as aves se adaptem ao alimento durante este período, garantindo assim, um bom desempenho futuro. As aves quando não alimentadas precocemente, não desenvolvem o máximo potencial do trato gastrintestinal, podendo causar redução do crescimento, redução da resistência a doenças e utilização ineficiente dos alimentos, proporcionando um baixo rendimento ao abate (Uni e Ferket, 2004).

Sendo assim, o objetivo do presente trabalho foi determinar a influência do fornecimento de diferentes quantidades de ração pré-inicial no desempenho, morfometria intestinal e peso dos órgãos do trato gastrointestinal de frangos de corte na fase inicial.

\section{MATERIAL E MÉTODOS}

Este trabalho foi realizado no Setor de Avicultura da Universidade Estadual do Oeste do Paraná UNIOESTE, Campus de Marechal Cândido Rondon - PR, em um galpão de alvenaria, com pé direito de 3,0 $\mathrm{m}$, contendo muretas laterais de $0,30 \mathrm{~m}$, cortinas, tela, cobertura com telhas de cerâmica francesa, provido de lanternim e subdividido em boxes com dimensão de $1,30 \times 1,35 \mathrm{~m}$. O piso de cimento foi recoberto com maravalha de pinus de primeiro uso, com espessura de $10 \mathrm{~cm}$.

O sistema de aquecimento foi realizado por meio de lâmpadas de infravermelho de $250 \mathrm{~W}$, sendo instalada uma lâmpada por boxe, cuja altura e acionamento foram regulados de acordo com a necessidade e crescimento das aves. O programa de iluminação utilizado foi o de 24 horas de luminosidade (luz natural + artificial). Quando necessário o resfriamento do ambiente foi utilizado ventiladores e nebulizadores.

Ao todo foram utilizados 576 pintos de corte, machos, da linhagem Cobb-500, no período de 1 a 21 dias de idade, com peso médio inicial de 44,25 $\pm 0,17 \mathrm{~g}$ adquiridos de incubatório comercial. A ração e a água foram fornecidas ad libitum durante todo o período experimental, sendo utilizados comedouros tubulares e bebedouros tipo nipple.

As aves foram distribuídas em um delineamento experimental inteiramente casualizado, com quatro tratamentos, que consistiram no fornecimento de diferentes quantidades de ração pré inicial (100, 200, 300 e $400 \mathrm{~g}$ ), com seis repetições e 24 aves por unidade experimental - UE.

Ao término do consumo (6, 8, 10, 12 dias) das respectivas quantidades de ração, as aves de cada UE passaram a receber uma ração inicial única, a base de milho e farelo de soja, conforme as recomendações de Rostagno et al. (2011), até os 21 dias de idade, quando foram então pesadas, bem como as sobras de ração para a determinação das variáveis de desempenho.

As variáveis de desempenho consumo médio de ração (CMR), ganho de peso (GP) e conversão alimentar (CA) foram determinados aos 21 dias de idade. $\mathrm{O}$ CMR foi calculado em gramas (g), pela diferença entre a ração fornecida e a sobra do comedouro, em cada UE. O GP, expresso em gramas, foi calculado como a diferença entre os pesos inicial e final das aves. A CA ( $g$ $\mathrm{g}^{-1}$ ) foi obtida dividindo-se o CMR pelo GPM das aves. A mortalidade foi observada diariamente para a realização das correções no consumo de ração e conversão alimentar, segundo Sakomura e Rostagno (2016).

Aos 21 dias de idade, seis aves de cada tratamento, com peso representativo (média $\pm 5 \%$ ), foram abatidas por deslocamento cervical, para determinação do peso relativo (\% do peso vivo) dos órgãos do trato gastrointestinal (proventrículo, moela, intestino delgado, pâncreas e fígado), comprimento do intestino delgado e colheita de fragmentos do jejuno para análise da morfometria intestinal por microscopia de luz. Para amostragem dos segmentos, considerou-se o jejuno, desde a porção distal da alça duodenal até o divertículo de Meckel.

Para as análises morfométricas, fragmentos de dois cm do segmento foram abertos longitudinalmente, lavados com solução salina, fixados em solução de formalina tamponada (10\%) em seguida desidratados em uma série de concentrações crescentes de alcoóis, diafanizados em xilol e incluídos em parafina (Luna, 1968). Após a microtomia semisseriada (cortes de sete $\mu \mathrm{m})$, os cortes foram corados pela técnica de hematoxilina e eosina.

As análises morfométricas (30 leituras/amostra) foram realizadas utilizando o sistema de imagens Motic Image Plus 2.0. A altura das vilosidades foi tomada a partir da região basal, que coincide com a porção superior das criptas até o ápice, e as criptas, da base até a região de transição vilo:cripta. A partir dos valores mensurados, foi calculado a relação altura de vilo:profundidade de cripta, dividindo-se o valor da altura do vilo pelo valor de profundidade de cripta.

Como procedimento estatístico, os resultados obtidos foram submetidos à análise de variância a 5\% de probabilidade e posterior regressão polinomial. As análises estatísticas foram realizadas utilizando o programa SAEG - Sistema para Análises Estatísticas e Genéticas.

\section{RESULTADOS E DISCUSSÃO}

O fornecimento de diferentes quantidades de ração pré-inicial não alterou $(\mathrm{P}>0,05)$ o peso médio $(\mathrm{PM})$, ganho de peso (GP) e consumo de ração (CR) aos 21 
Tabela I. Desempenho de frangos de corte aos 21 dias de idade alimentados na fase pré-inicial com diferentes quantidades de rações (Performance of broiler chickens at 21 days of age fed pre-starter with different amounts of feed).

\begin{tabular}{lcccc}
\hline $\begin{array}{l}\text { Ração pré-inicial } \\
\text { (g/ave) }\end{array}$ & PM $(\mathrm{g})$ & $\mathrm{GP}(\mathrm{g})$ & $\mathrm{CR}(\mathrm{g})$ & $\mathrm{CA}$ \\
100 & 918,05 & 873,83 & 1192,15 & 1,366 \\
200 & 916,97 & 871,68 & 1236,44 & 1,418 \\
300 & 916,36 & 872,12 & 1262,68 & 1,448 \\
400 & 911,11 & 866,82 & 1241,42 & 1,433 \\
CV (\%) & 4,02 & 4,23 & 3,43 & 3,30 \\
P regressão & - & - & - & $0,035(\mathrm{~L})$ \\
\hline
\end{tabular}

Equação de regressão polinomial

${ }^{1} C A=1,359167+0,00023 X\left(R^{2}=0,70\right)$

Peso médio (PM), ganho de peso (GP), consumo de ração (CR) e conversão alimentar (CA); Coeficiente de variação (CV); Linear (L).

de idade das aves (Tabela I). No entanto, houve efeito linear crescente $(\mathrm{P}<0,05)$ para conversão alimentar (CA), havendo piora na conversão conforme aumento da quantidade de ração pré-inicial fornecida.

Estudos demonstram resultados heterogêneos quanto aos benefícios do fornecimento de diferentes quantidades de ração pré-inicial para frangos de corte. Stringhini et al. (2003) ao avaliarem aves aos 21 dias, criadas com períodos de 7, 10 e 14 dias de fornecimento da ração pré-inicial, não verificaram diferenças quanto ao CR e CA. Em contrapartida, Silva et al. (2004) observaram um maior ganho de peso para as aves que ingeriram 300 gramas de ração pré-inicial, quando comparadas as que receberam 100 e 200 gramas. Gomes et al. (2008) constataram que aves alimentadas com dieta pré-inicial por um período de sete dias apresentaram maior consumo e ganho de peso aos 42 dias, em comparação àquelas que não receberam a dieta pré-inicial. No entanto, relataram que as aves quando alimentadas com dieta pré-inicial até os 14 dias, apresentaram GP e CA inferiores nos primeiros 21 dias, o que pode estar relacionado a maior quantidade de nutrientes da dieta. Estes resultados enfatizam a importância do fornecimento de uma dieta diferenciada nos primeiros dias de vida da ave, favorecendo o aproveitamento dos nutrientes com consequente influência sobre o desempenho final. Neste contexto, Lilburn (1998) recomenda que essa dieta pré-inicial seja utilizada nos primeiros sete ou 10 dias de vida das aves, devendo ser considerada como um investimento e não um custo no sistema de produção.

No presente trabalho houve uma melhor CA nos animais que receberam a menor quantidade de ração pré inicial, demonstrando um melhor aproveitamento dos nutrientes. Com o aumento da quantidade de ração pré-inicial fornecida, houve piora na conversão, indicando que o excesso de nutrientes na dieta, como por exemplo, aminoácidos, pode comprometer a produção, devido ao gasto de energia requerido no metabolismo e excreção destes compostos (Araújo et al. 2002). Tais resultados diferem dos observados por Silva et al. (2004) que encontrara os melhores resultados para conversão aos 21 dias de idade para as aves que consumiram 200 e $300 \mathrm{~g}$ de dieta pré-inicial.

O fornecimento de alimento precoce estimula o desenvolvimento do sistema termorregulatório, além do sistema imune e digestório (Van Den Brand et al., 2010) e a quantidade de nutrientes que a ave recebe possui relação direta com o crescimento inicial, (Yang et al., 2009).

O peso relativo do proventrículo, moela, intestino delgado, fígado, pâncreas e o comprimento do intestino delgado dos frangos aos 21 dias de idade não foram alterados $(\mathrm{P}>0,05)$ pelo fornecimento de diferentes quantidades de ração pré-inicial (Tabela II). As diferentes quantidades de ração pré-inicial fornecidas as aves também não influenciaram $(\mathrm{P}>0,05)$ a altura de vilosidade, profundidade de cripta e relação vilo:cripta no jejuno aos 21 dias de idade.

A determinação do peso relativo dos órgãos do trato gastrointestinal na fase pré-inicial é de fundamental importância para caracterizar o bom desenvolvimento da ave, fornecendo suporte anatomo fisiológico para os processos digestivos e absortivos de nutrientes, influenciando diretamente o desempenho das aves. Desta forma, os resultados obtidos indicam que as diferentes quantidades de ração pré-inicial fornecida às aves não interferiram no desenvolvimento dos órgãos do trato gastrointestinal e no comprimento do intestino delgado, permitindo um desempenho apropriado nesta fase.

Em contrapartida, em estudo realizado por Nakage (2007) foi verificado que o fornecimento tardio de ração e água na fase inicial comprometeu o desenvolvimento do sistema digestório das aves, causando uma menor atividade enzimática e menor desenvolvimento dos órgãos (Oliveira, 2012).

Segundo Freitas et al. (2007) a maior altura das vilosidades está relacionada aos resultados de desempenho, em que as aves apresentam maior GP e melhor CA. Isso está relacionado com a integridade da mucosa intestinal e processo metabólico, que confere a característica de quanto maior o tamanho dos vilos, maior é a capacidade de digestão e absorção de nutrientes, em função da maior área de contato e efetividade enzimática no nível de mucosa e lúmen intestinal. Sendo assim, independentemente da quantidade de ração pré-inicial fornecida as aves, o desenvolvimento do trato gastrointestinal como um todo se apresentou de forma adequada, proporcionando um desempenho satisfatório em todos os tratamentos.

Práticas nutricionais que atendam as exigências na primeira semana de vida da ave favorecem o desenvolvimento do trato gastrointestinal e garantem um bom desempenho nas próximas fases (Gentilini et al., 2013; dos Santos et al., 2013). O fornecimento de ração o mais breve possível após a eclosão estimula a secreção de enzimas que são dependentes de substrato, favorecendo a digestão e absorção dos nutrientes. Deve-se 
Tabela II. Peso relativo (\%) dos órgãos do trato gastrointestinal, comprimento do intestino delgado (cm), altura de vilo $(\mu \mathrm{m})$, profundidade de cripta $(\mu \mathrm{m})$ e relação altura de vilo:profundidade de cripta do segmento jejuno de frangos de corte aos 21 dias alimentados com diferentes quantidades de ração pré-inicial. (Relative weight $(\%)$ of organs of the gastrointestinal tract, length of small intestine $(\mathrm{cm})$, height of villi $(\mu \mathrm{m})$, depth of crypt $(\mu \mathrm{m})$ and height of villi ratio: crypt depth of the jejunum segment of broilers at 21 days fed with different amounts of pre-starter feed).

\begin{tabular}{lccccccccc}
\hline $\begin{array}{l}\text { Ração } \\
\text { pré-inicial (g) }\end{array}$ & Proventrículo & Moela & IntD & CID & Fígado & Pâncreas & $\begin{array}{c}\text { Altura de } \\
\text { Vilo }\end{array}$ & $\begin{array}{c}\text { Profundida- } \\
\text { de de Cripta }\end{array}$ & Vilo:Cripta \\
100 & 0,523 & 2,021 & 7,340 & 139 & 2,858 & 0,395 & 647,95 & 67,13 & 9,64 \\
200 & 0,593 & 2,281 & 7,988 & 148 & 2,713 & 0,418 & 572,30 & 64,95 & 8,93 \\
300 & 0,533 & 2,315 & 7,796 & 132 & 2,598 & 0,410 & 550,97 & 52,42 & 11,44 \\
400 & 0,546 & 2,126 & 7,748 & 139 & 2,456 & 0,391 & 589,15 & 58,39 & 10,20 \\
CV (\%) & 11,64 & 12,21 & 17,87 & 8,60 & 12,73 & 15,93 & 19,05 & 22,85 & 19,32 \\
\hline
\end{tabular}

IntD: Intestino delgado; CID: Comprimento intestino delgado; CV= Coeficiente de variação.

considerar tambem, que a qualidade dos ingredientes da ração é tão importante quanto a disponibilidade de alimento.

\section{CONCLUSÃO}

O fornecimento de 100 gramas de ração pré-inicial proporcionou melhor conversão alimentar aos 21 dias de idade, sem interferir no desenvolvimento do trato gastrointestinal das aves.

\section{BIBLIOGRAFÍA}

Araújo, L.F, Junqueira, O.M, Araújo, C.S.S 2002, Different criterion of feed formulation for broilers in the period 1 to 21 days of age, Brazilian Journal of Poultry Science, vol.4, pp.195-202.

Gentilini, F.P, Anciuti, M.A, Pereira, L.M.R 2013, Tópicos atuais na produção de suínos e aves. Pelotas: Instituto Federal Sul-rio-grandense, $271 \mathrm{p}$.

Gomes, G.A, Araújo, L.F, Prezzi, J.A, Savietto, D, Júnior, J.R.S, Valério, J 2008, Tempo de fornecimento da dieta pré-inicial para frangos de corte com diferentes pesos ao alojamento, Revista Brasileira de Zootecnia, vol.37, no.10, pp.1802-1807.

Lilburn, M.S 1998, Pratical aspects of early nutrition for poultry, Journal Applied Poultry Research, vol. 07, no. 4, pp. 420-424.

Luna, G. C 1968, Manual of histologic staining methods of the armed forces. Institut of pathology. 3.ed. New York: Mc Graw-Hill, 285p.

Nakage, E. S. Respostas fisiológicas de pintos submetidos a diferentes períodos de jejum: parâmetros hematológicos e intestinais. 2007. 100 f. Tese (Doutorado em Zootecnia) - Faculdade de Ciências Agrárias e Veterinárias, Universidade Estadual Paulista, Jaboticabal. 2007.
Noy, Y, Sklan, D 1998, Yolk utilization in the newly hatched poultry, British Poultry Science, vol. 39, pp. 446-451.

Oliveira, C.E.C. Efeito do jejum alimentar durante a janela de nascimento em frangos. 2012. 45 f. Dissertação (Mestrado em Ciências Veterinárias) - Universidade Federal do Paraná, Curitiba. 2012.

Prabakar, G., Pavulrai, S., Shanmuganathan, S., Kirubakaran, A., \& Mohana, N, 2016, Early nutrition and its importance in poultry: a review. Indian Journal of Animal Nutrition, vol.33, no.3, pp.245-252.

Rostagno, H.S, Albino, L.F.T, Donzele, J. L, Gomes, P. C, Oliveira, R. F, Lopes, D. C, Ferreira, A. S, Barreto, S. L. T, 2011 Tabelas brasileiras para aves e suínos: Composição de alimentos e exigências nutricionais. Viçosa:UFV, Departamento de Zootecnia, 2 ed. 186p.

Sakomura, N. K, \& Rostagno, H, S 2016, Métodos de pesquisa em nutrição de monogástricos. -2. ed. - Jaboticabal: Funep. 262p.

dos Santos, F. R, Oliveira, P. R, Minafra, C. S, Duarte, E. F, de Almeida, R. $R, \&$ da Silva, W. J 2016, Desenvolvimento digestivo e aproveitamento energético em frangos de corte, PUBVET, vol. 6, no. 18, pp. 1369-1374.

Silva, J.R.L, Rabello, C.B.V, Dutra Júnior, W.M, Ludke, M.C.M.M, Barroso, J.F, Freitas, C.R.G, Campleo Filho, E.V.B, Aquino, L.M 2004, Efeito da forma física e do programa alimentar na fase pré-inicial sobre desempenho e características de carcaça de frangos de corte, Acta Scientarum Animal Science, vol.26, no.4, pp.543-551.

Uni, Z, Tako, E, Gal-Garber, O, Sklan, D 2003. Morphological, Molecular, and Functional Changes in the Chicken Small Intestine of the Late-Term Embryo, Poultry Science, vol. 82, no. 6, pp. 1747-1754.

Uni, Z, Ferket, R. P 2004, Methods for early nutrition and their potential, Poultry Science, vol. 60, no. 1, pp.101-111.

Van Den Brand, H, Molenaar, R, Van Der Star, I, \& Meijerhof, R 2010, Early feeding affects resistance against cold exposure in young broiler chickens, Poultry Science, vol. 89, no. 9, pp. 716-720.

Yang, H, Wang, Z, Shi, S, Lu, J, \& Li, W 2009, Effects of starter feeding time on body growth and viscera development of newly hatched chicks, Italian Journal of Animal Science, vol. 8, pp. 585- 593. 